\title{
Nasotracheal intubation for airway management during anesthesia
}

\section{Dong Ho Park, Chia An Lee, Chang Young Jeong, and Hong-Seuk Yang}

Department of Anesthesiology and Pain Medicine, Daejeon Eulji University Medical Center, Eulji University, School of Medicine, Daejeon, Korea
Received April 20, 2021

Revised July 6, 2021

Accepted July 7, 2021

\section{Corresponding author}

Hong-Seuk Yang, M.D., Ph. D.

Department of Anesthesiology and

Pain Medicine, Daejeon Eulji

University Medical Center, Eulji

University, School of Medicine, 95

Dunsanseo-ro, Seo-gu, Daejeon

35233, Korea

Tel: 82-42-611-3883

Fax: 82-42-259-1111

E-mail: hsyang@amc.seoul.kr

\begin{abstract}
Nasotracheal intubation is used as a basic method for airway management, along with orotracheal intubation under anesthesia and intensive care. It has become an effective alternative method to orotracheal intubation with increased benefits of offering better mobility and surgical field in oral and maxillofacial surgery and possibly in trauma and critically ill patients. Nasotracheal intubation is performed through a relatively narrow nasal cavity; therefore, additional precautions are needed. Accordingly, nasotracheal intubation methods have evolved over the years with accumulated clinical experience and improved instruments to facilitate safe intubation with reduced complications. Therefore, in this review article, we summarize the basic anatomy of the nasal airways to clarify the precautions, delineate the history and development of various methods and instruments, and describe the indications, contraindications, complications, and preventive methods of nasotracheal intubation.
\end{abstract}

Keywords: Airway management; Anesthesia; Endotracheal intubation; Nasal cavity.

\section{INTRODUCTION}

Airway management plays an important role in the management of critically ill patients, trauma patients, and anesthesia for all surgeries and procedures performed inside and outside of the operating room. From this point of view, anesthesiologists choose and apply various methods such as the bag and mask methods, airways simply inserted into the oral or nasal cavity, supraglottic airway devices, oral or nasotracheal intubation, percutaneous dilated cricothyroidotomy, and tracheostomy according to the patient's condition and the need for surgery. Among these methods, supraglottic airway devices or orotracheal intubation are usually selected. Nasotracheal intubation is attempted for head and neck surgery, oral surgery, or for keeping the airway safe while preventing further damage in some trauma patients [1-3].

Nasotracheal intubation is a common airway manage- ment method used for anesthesia. Since the endotracheal tube is inserted into the trachea through the nasal cavity, it is easier to fix and stabilize through the small diameter of the nasal pathway compared to the oral cavity. It is the preferred method not only for anesthesiologists but also for surgeons who perform surgeries in the head and neck region, especially oropharyngeal, dental, and maxillofacial surgeries, as it helps to improve vision and access for surgery [3-5].

In the nasotracheal intubation process and management, additional instruments, drugs, and skilled maneuvers are required, and with recent developments in techniques and methods, potential problems or complications arising from the blind introduction of the endotracheal tube into the nasal cavity can be avoided [6-9]. Therefore, this study aimed to summarize the latest findings with a literature review regarding nasotracheal intubation. It will include the newly introduced information along with methods that have been

This is an Open Access article distributed under the terms of the Creative Commons Attribution Non-Commercial License (http://creativecommons.org/licenses/by-nc/4.0) which permits unrestricted non-commercial use, distribution, and reproduction in any medium, provided the original work is properly cited.

Copyright (c) the Korean Society of Anesthesiologists, 2021 
used in clinical practice so far, associated precautions, indications and contraindications, various complications such as epistaxis and sinusitis, and prevention methods.

\section{HISTORY AND DEVELOPMENT}

Endotracheal intubation is applied to more than $80 \%$ of patients requiring general anesthesia and has been in use for more than 1000 years [10]. Alfred Kirstein introduced direct laryngoscopy in 1895, and Franz Kuhn performed endotracheal intubation to maintain the airway in patients intoxicated with morphine and introduced the nasal route. Guedel and Waters added cuffs to the tubes in 1932 to ensure positive pressure ventilation $[10,11]$.

Nasotracheal intubation was introduced by Franz Kuhn in 1902, suggesting that it would be more physiological than orotracheal intubation. Magill introduced the oral airway or short rubber tube through the nasal cavity to the pharynx to insufflate anesthetics and oxygen on a hospital ship during World War I in 1919. Nasotracheal intubation was attempted to compensate for these shortcomings. However, since there were no adjuvant devices such as a laryngoscope or Magill forceps at this time, various postures (e.g., sitting positions) were attempted according to the skill and preference of the anesthesia providers. A "blind nasal intubation" was performed by inserting a catheter, expiratory tube, or a tube made of a soft material causing relatively little stimulation to the breathing apparatus [12,13]. It was introduced as an effective method when Rowbothan first attempted oral surgery in the operating room in 1920 [11-14]. At the same time, nasotracheal intubation was introduced as an airway management method that could be effectively applied for longterm care in the intensive care unit, but there was a risk of infections such as sinusitis [15].

In the past, various postures for orotracheal and nasotracheal intubation were applied according to individual technical preferences. Magill first described a sniffing position with the head lifted approximately $15^{\circ}$ from a stable supine position in 1936 [13]. Reports on nasotracheal intubation using a fiberoptic bronchoscope began in the 1960s. Instruments such as the video-laryngoscope and Magill forceps, improvement of endotracheal tube materials, and application of vasoconstrictors (e.g., epinephrine and phenylephrine) and lubricants helped facilitate nasotracheal intubation, thereby increasing its success rate [16].

\section{ANATOMY}

The anatomical characteristics of the airway from the nostril to the trachea, where the tube passes through while attempting nasotracheal intubation, can be described as follows: The nasal cavity refers to the area starting from the anterior nares (nostrils), through the posterior end of the nasal septum, and then through the posterior nasal apertures (choanae) to the nasopharynx in contact with the oropharynx. The skull base and intracranial components are located at the top, and the hard palate in contact with the oral cavity is located at the bottom. The medial wall of the nasal cavity is formed by the nasal septum, which consists of cartilage in the front and bone in the rear. The bony part on the medial surface is connected to the perpendicular plate of the ethmoid bone at the top and the vomer at the bottom [2-5]. The lateral surface is connected to the medial surface of the orbit at the top and the medial wall of the maxillary sinus at the bottom. On the lateral surface, three nasal conchae (turbinate) are curved downward and are composed of bone covered with a thick respiratory mucosa [2-5].

The inferior turbinate is the largest of the three turbinates on both the left and right sides and is firmly fixed on the outer surface, connected to the conchal crest of the maxilla, the descending process of the lacrimal bone, the frontal process of the maxilla, the uncinate process of the ethmoid, and the conchal crest of the palatine bone. If avulsion occurs during nasotracheal intubation despite this tight fixation, the inferior turbinate may be fractured and enter the nasotracheal tube, enter the main bronchus causing an obstruction. The inferior turbinate can be easily observed using a rhinoscope from the outside, but it is difficult to distinguish it from nasal polyps [2-7]. The superior turbinate was the smallest and was located just above and behind the middle turbinate. In addition, it is attached to the skull base upward and the nasal wall on the side [2-6]. The middle turbinate is an integral part of the ethmoid bone, which is loosely attached to the free, convoluted margin of the thin lamella descending from the cribriform plate and the posterior ethmoid air cells in the back. It is mainly responsible for olfaction, humidification, and lubrication, mediated via the olfactory nerve, in the cribriform plate, and controls temperature and humidity in the inhaled air while performing filtration [2-6].

Each turbinate was covered by a thick mucous membrane, and blood was supplied from the anterior ethmoidal artery. The lamina propria of the mucous membrane contains cavernous sinusoids or plexus of large veins and is very vulnera- 
ble to trauma. Situations such as traumatic avulsion can lead to massive epistaxis, and excessive force on the turbinate can lead to olfactory nerve injury or fracture of the cribriform plate, which may be accompanied by cerebrospinal fluid (CSF) rhinorrhea [2-6,17].

The upper part of the nasal cavity is narrow and connected with the cribriform plate of the ethmoid, with the lateral wall and septum, distinguishing the nasal cavity from the anterior cranial fossa. The nasal cavity is entirely covered by a respiratory mucosa composed of ciliated pseudostratified columnar epithelium, with many mucous secretions for humidification and entrapment of inhaled impurities [2-7].

The sphenopalatine artery from the maxillary artery is the main blood supply to the nasal cavity. The Kiesselbach's plexus is formed by anastomosis with the ascending branch of the greater palatine artery and superior labial artery in the anterior part of the nasal septum (Little's area). In the nasal cavity, mucous membranes and blood vessels play an important role, but they are the main sites of epistaxis that occur naturally and can easily lead to bleeding even with light external trauma [2-7].

Internal structural abnormalities of the nasal cavity are often asymmetric. The most common anatomical abnormality was septal deviation (SD). It is commonly found in the anterior cartilaginous part and rarely in the septum, which consists of bone in the rear. It often occurs due to trauma. However, it can also occur without any specific injury due to fetal intrauterine molding or trauma during parturition. In addition, nasal SD may occur due to maldevelopment of the vomeropalatal complex [18]. SD causes a change in airflow, resulting in a change in the mucosal lining. Dryness and ulceration of the mucous membrane and hypertrophy may occur in the convex and concave parts of the inferior turbinate, respectively. These SDs often result in the narrowing of the nasal airway. Severe structural abnormalities are rare but may occur after shock or surgery. Although rare, choanal atresia is a congenital malformation that may occur during childhood $[5,6]$.

In children, it generally takes eight years to develop an airway similar to that of an adult. Because the length of the trachea is relatively short, the change in length according to the flexion and extension of the neck is large, and a tube may be inserted deeply or fall out depending on the flexion and extension of the neck $[19,20]$. Infants and young children are susceptible to upper respiratory tract obstruction and respiratory failure. Because the length of the trachea is relatively short in children, the endotracheal tube may be displaced into either the endobronchial or cephalad position towards the glottis, according to slight postural changes in flexion or extension of the neck. Airway resistance is increased owing to the selection of small tubes for a relatively smaller airway. Therefore, it should be selected and attempted after understanding the characteristics of the pediatric airway depending on the age and confirming the diameter, length, and characteristics of the endotracheal tube [20].

\section{NASOTRACHEAL INTUBATION}

\section{Preanesthetic evaluation}

For each patient requiring anesthesia, anesthesiologists should perform the pre-anesthetic evaluation required for nasotracheal intubation, along with preoperative evaluation.

1. During patient interviews and examinations, the presence or absence of a history of epistaxis, condition of teeth, potential anesthetic problems such as a history of injuries to the face, and potential complications or contraindications to the planned procedure should be identified (e.g., airway disorders, progressive infections including sinusitis, swelling, epistaxis, etc.). In addition, a history of anaphylaxis and allergic reactions to drugs, including drugs used for anesthesia, should be elicited and reviewed. Furthermore, the risks of anesthesia should be based on the patient history and family history of previous surgery and anesthesia (e.g., complications from anesthesia, malignant hyperthermia, etc.) and established standards (e.g., American Society of Anesthesiologists classification of risk), and identifying the need for additional medical consultations and tests in the pre-anesthetic evaluation. Finally, a plan should be established for anesthesia. The induction, maintenance, recovery of anesthesia, postoperative management, and risks and benefits of anesthesia should be discussed with the patient and caregiver.

2. The problems associated with airway management and nasotracheal intubation should be tested and identified (e.g., presence of dentures or loose teeth, limitation of mouth opening, jaw protrusion, length and thickness of the neck, range of motion of the head/neck, etc.), and additional tests such as endoscopy, X-ray, computerized tomography (CT), and magnetic resonance imaging (MRI) should be performed to identify the problems in nasotracheal intubation and prepare for alternative methods. Nasotracheal intubation should be performed based on the overall results of preoperative evaluation. If nasotracheal 
intubation is expected to be difficult, a discussion should be conducted with the surgeon to decide whether to perform nasotracheal intubation or not and to devise alternative methods [21,22]. The pre-anesthetic evaluation for these patients should be performed within $48 \mathrm{~h}$ before surgery and should be documented and recorded [2-6].

\section{Considerations for nasotracheal intubation}

\section{Selection of the position of the nostril for intubation}

The supine position is mostly preferred to perform the orotracheal intubation. However, blind nasotracheal intubation can be performed in the sitting position, depending on the skill of the operator [1-4]. Selecting the nostril to use for endotracheal intubation is an important decision to consider for facilitating intubation and reducing complications. In the pre-anesthetic evaluation, the method of blocking one nostril and breathing on the other side and selecting the comfortable side between them may be the easiest to apply.

However, as described above, it may be determined according to the shape of the nasal septum by preoperative examination. Although it is possible to select the nostril in most patients using anterior rhinoscopy, it is difficult to accurately determine any abnormalities in the posterior nasal cavity. Nasal endoscopy helps select nostrils for endotracheal intubation and for evaluating intranasal abnormalities. Flexible endoscopy also allows for detailed examination of the entire nasal cavity for nostril selection [2-4]. Depending on the patient's condition, methods such as facial radiography, CT, and MRI can be used to examine anatomical and pathological abnormalities such as SD [23,24].

\section{Selection of the lower and middle regions of the inferior turbinate in the nasal cavity}

In nasotracheal intubation, there are lower and upper pathways for passing through the nasal cavity, with the inferior turbinate at the center. The middle turbinate is a thin lamella that is attached to the base of the cribriform plate and is rich in blood vessels. Trauma such as avulsion of the middle turbinate may result in massive epistaxis, CSF rhinorrhea, or damage to the olfactory nerves distributed in the cribriform plate. The lower pathway is the pathway between the floor of the nose and the inferior turbinate. The upper pathway is the pathway between the inferior turbinate and the middle turbinate. Thus, the lower pathway is safer because it avoids trauma to the middle turbinate and cribriform plate $[9,17]$.

\section{Procedures of nasotracheal intubation}

After all the tests related to surgery and anesthesia were completed, the occurrence of complications during nasotracheal intubation should be reduced. The patient who enters the operating room should be first identified and equipped with monitoring instruments for blood pressure, electrocardiogram, peripheral oxygen saturation $\left(\mathrm{SpO}_{2}\right)$, and end-tidal carbon dioxide tension $\left(\mathrm{ETCO}_{2}\right)$. Subsequently, management procedures for nasotracheal intubation should be performed.

\section{Premedication}

Despite the individual differences, sedation or analgesia may be necessary for most patients, as they may exhibit anxiety, particularly if awake intubation is planned. Therefore, drugs such as opioids (e.g., fentanyl, alfentanil, etc.) and sedatives (e.g., benzodiazepine and dexmedetomidine) may be selected depending on the patient's condition and post-intubation management.

\section{Preparation of the nasal mucosa}

Commonly used drugs for lubrication and vasoconstriction include lidocaine spray or jelly. It is sprayed at the time of inspiration and enters the glottis to exhibit an effect.

1) Transtracheal/translaryngeal anesthesia: After checking the cricothyroid membrane between the thyroid and cricoid cartilages with an index finger, a $23 \mathrm{G}$ needle was used with the opposite hand to puncture and aspirate air to check the lumen of the larynx; lidocaine (2\%, approximately $2 \mathrm{ml}$ ) was injected, and the syringe was removed immediately. The patient may cough during the procedure, and the local anesthetic can provide the effects of applying anesthesia from the epiglottis site to the carina [9]. Transtracheal/translaryngeal anesthesia is preferred for non-general anesthesia and is not necessarily required for general anesthesia [25,26].

2) Vasoconstrictor: In most cases, lidocaine (3-4\%) jelly with phenylephrine $(0.05-1 \%)$ or epinephrine $(1: 200,000)$ added, or oxymetazoline is used to induce vasoconstriction [4-6].

3) Anti-sialagogues: Before using the airway device, anti-sialagogues (e.g., glycopyrrolate $0.4 \mathrm{mg}$ intramuscularly [IM] or intravenously [IV] / atropine 0.5 - $1 \mathrm{mg}$ IM or IV) are used. Excessive secretions can make it difficult to view the laryngoscope and interfere with the effective penetration of local anesthetics into the mucous membrane [2-6]. 
4) Lubrication of endotracheal tube: Before insertion through the nostril, sufficient lubricant is applied so that the tube can smoothly pass through the relatively narrow nasal cavity. The lubricant is water-soluble, and the addition of local anesthetics such as lidocaine would increase the analgesic effect, while the addition of vasoconstrictors would reduce the risk of bleeding [2-6].

\section{Oxygenation}

In the entire process of nasotracheal intubation, preoxygenation should be performed with $100 \%$ oxygen to the patient before proceeding with the procedure. This increases the body oxygen reserve and delays arterial hemoglobin desaturation because an unexpected situation may arise, resulting in hypoxia, hypoventilation, and loss of airway patency.

Preoxygenation is recommended to ensure that the patient's end-tidal oxygen concentration is 0.9 or more or the end-tidal nitrogen concentration is $5 \%$ or less by tidal breathing [27-29]. Preoxygenation methods include tidal volume $\times 3$ min using a $100 \%$ oxygen flow, four deep breaths (vital capacity) within $30 \mathrm{~s}$ using a $100 \%$ oxygen flow, eight deep breaths within one min using a $100 \%$ oxygen flow [27], or the transnasal humidified rapid-insufflation ventilation exchange using the Optiflow high-flow humidified oxygen delivery system [30-32].

\section{Anesthesia}

Nasotracheal intubation is attempted under general anesthesia with intravenous anesthetic agents or inhaled anesthetics with prior sufficient preoxygenation. Neuromuscular blockers are usually administered after the loss of consciousness for endotracheal intubation under general anesthesia, unless contraindicated or not otherwise required. If neuromuscular blockers are not used for nasotracheal intubation, particular attention should be paid to reduce patient movement, airway irritation, and sympathetic stimulation, because the injury may be invoked during the intubation process [33-35].

\section{Insertion through nasal cavity for nasotracheal intubation}

The procedure of nasotracheal intubation can be explained by dividing it into three phases: 1) passage through the nose into the pharynx, 2) laryngoscope-guided passage into the glottic inlet, and 3) laryngoscope-guided passage into the trachea [36].

The first phase is explained as follows. An endotracheal tube of appropriate size was selected for the patient, and a lubricant was applied to the site in preparation for insertion. The endotracheal tube was inserted slowly along the bottom of the nasal cavity by pushing it down to the rear through the selected nostril and treated with local anesthesia and vasoconstrictor. While avoiding excessive force, the endotracheal tube should be inserted by rotating it slowly [2-5]. While advancing from the nasal cavity to the oropharynx during the intubation process, resistance may be felt upon passing through the nasopharyngeal junctional space and the posterior nasopharynx. In this case, the endotracheal tube should be inserted while rotating or extending the patient's neck $[37,38]$.

\section{Nasotracheal intubation after insertion through the nasal cavity}

In the subsequent process, there may be differences in the insertion method depending on the preference of the anesthesiologist and the patient's condition. The basic methods are described in this section. In the author's clinical experience, sometimes more than one attempt of nasotracheal intubation is required in cases of a difficult airway. In such cases, the endotracheal tube inserted into the nasal cavity must be removed and bag-mask ventilation is reinstituted while preparing for the next intubation trial. Multiple nasotracheal intubation attempts could hinder subsequent bagmask ventilation and/or intubation by obscuring the laryngeal view with nasal bleeding and secretions. Therefore, it is necessary to accurately determine the patient's condition, the degree of difficulty in intubation, and plan to succeed at the first intubation attempt. It is also imperative to prepare adjuvant devices, such as emergency airways and suction devices [39].

1) Blind nasal intubation: Blind nasal intubation is the method used in the past when there is no adjuvant device, in which an endotracheal tube of an appropriate size is selected and prepared by applying a lubricant for insertion. The unanesthetized nostril and lip were blocked, while the chin of the patient was held by the left hand. The endotracheal tube was inserted slowly along the bottom of the nasal cavity by pushing it down to the rear through the anesthetized nostril. While observing the breathing sounds, if the tube is inserted into the pharynx and placed in the middle, it passes under the epiglottis, passes through the vocal cords where the reflex is temporarily stopped by local anesthesia, and is inserted into the larynx. Closure of the glottis can be prevented if the gag reflex 
and involuntary swallowing are blocked by appropriate local anesthesia. In conscious patients, pharyngeal muscle tone may help with tracheal intubation. This leads to the endotracheal tube below the epiglottis and into the space between the vocal cords. Endotracheal intubation is mostly performed in the supine position, but in rare cases where intubation is difficult, it may be more effective to perform this in a sitting position [40]. Blind nasal intubation was selected when direct laryngoscopy was difficult or impossible. In recent years, with the advent of flexible fiberoptic endoscopy, which is referred to as the "gold standard technique," blind nasal intubation has become an old manual technique [41].

2) Nasal intubation using laryngoscope and Magill forceps: Magill forceps were first introduced by Magill in 1920 to guide the nasally inserted endotracheal tube to pass through the glottis under direct or video-laryngoscope visualization $[42,43]$. When using a video-laryngoscope, there may be some inconvenience due to a lack of skills in the process of intubation while holding the tube with Magill forceps and viewing the video screen $[42,43]$. If it is difficult to insert the laryngoscope directly through the oral cavity, such as in patients with certain maxillofacial problems, submental intubation may be considered [44].

3) Fiberoptic nasal intubation: Nasotracheal intubation using a fiberoptic endoscope is similar to oral fiberoptic endoscopic intubation. While performing fiberoptic endoscopy, the path through which the endotracheal tube passes through the nasal cavity should be checked in advance. Intubation should proceed to the trachea, and the endotracheal tube should advance through it. However, if the tip of the fiberoptic endoscope is not sufficiently inserted into the trachea, the direction of insertion of the endotracheal tube may deviate. It may be deviated mainly by the epiglottis, arytenoid cartilage, pyriform fossa, or esophagus during insertion. It is possible to insert the endotracheal tube while rotating counterclockwise. In addition, examiners should be careful because the pyriform sinus may look like the glottis under the fiberoptic endoscope $[16,45]$.

Similar to the method of mounting a face mask while applying a fiberoptic endoscope, there is also a method of performing endotracheal intubation; by placing the little finger of one hand at the angle of the mandible, the ring finger at the body of the mandible, the middle finger under the mentum to lift the chin to maintain airway patency and improve the endoscopic view, and by using the thumb and index finger of the same hand to adjust the direction of the fiberoptic endoscope. Flexible fiberoptic intubation is preferred as the main technique for difficult intubation, but it has disadvantages in that it takes time and requires additional equipment as well as skilled maneuvers. However, it facilitates intubation in cases of difficult intubation, avoids damage to surrounding tissues and teeth, improves the view of the larynx, allows the easy decision of the nostril pathway, and causes less bleeding $[16,45]$.

The nasal trumpet may be preferred as it can facilitate intubation or insertion of a fiberoptic endoscope by increasing the diameter of the nostril. However, it cannot be removed after endotracheal intubation and must be held together until extubation. However, this method maintains the airway in patients whose trachea cannot be intubated or who need continuous airway management and allows mask ventilation and positive pressure ventilation. It can be also inserted into a patient with spontaneous breathing, awake, or under anesthesia. This may be ideal for maintaining the patient's airway during fiberoptic nasotracheal intubation. It is particularly useful in patients who require awake endotracheal intubation [46].

4) Nasotracheal intubation using a retromolar fiberscope: This method is generally referred to as "Bonfils" and uses a rigid fiberoptic endoscope that is useful in difficult airway management [47]. A tube of appropriate size was inserted into the nasal cavity and advanced until it reached the oropharynx. With the mandible pulled upward, the Bonfils endoscope was inserted into the oral cavity and advanced until the epiglottis and vocal cords were identified. After checking the position of the tracheal tube in the oropharynx, the tube was moved into the field of view of the Bonfils and inserted into the trachea. It requires a high level of skill and Magill forceps may be used. It may apply to patients with the immobilized cervical spine and significantly limited inter-incisor distance [47,48].

5) Use of lightwand (lighted stylet): A lightwand can be applied in the same way as in orotracheal intubation, but it should be inserted through the nasal cavity within the endotracheal tube. Therefore, it is difficult to apply this method with no flexibility. After the appropriate tube was inserted into the oropharynx, the lights in the operating room were dimmed, and a flexible lightwand was inserted into the tube by controlling the light rays appearing through the neck until it was seen directly above the cricothyroid membrane at the centerline of the neck. After that, the lightwand was fixed with one hand, and the tube was pushed with the other hand and inserted into the trachea. 
The lightwand is supplied in various forms, but traumatic events such as airway damage can be prevented by selecting a flexible lightwand suitable for the patient's condition and the skill level of the anesthesiologist $[49,50]$.

6) Nasotracheal intubation using endotracheal tube introducer (bougie): One or two endotracheal tube introducers can be used when orotracheal intubation is performed. Nasotracheal intubation can also be performed by applying one or two introducers. When using dual introducers, a cuffed endotracheal tube is orally intubated into the trachea, and a cuffed tube with an appropriate size and shape for nasotracheal intubation is inserted into the nasal cavity before proceeding to the oropharynx. The endotracheal tube introducer was then inserted into the glottis through the nasal tube, and the laryngoscope and Magill forceps were used to access the trachea. After deflating the cuff of the orotracheal tube, the introducer was advanced into the trachea and placed in line with the orotracheal tube. After advancing the introducer already mounted with the nasotracheal tube into the trachea along the orotracheal tube using a laryngoscope and Magill forceps, the orotracheal tube was removed, and a nasotracheal tube was inserted. Nasotracheal intubation was completed by removing the introducer in the nasotracheal tube and checking the location of the tube. The endotracheal tube introducer enables nasal intubation using a video-laryngoscope with the Seldinger technique in children [51]. Alternatively, the orotracheal tube should be removed by splitting, and the introducer may be inserted into the endotracheal tube through the nose. The tube was inserted through the endotracheal tube, after which it was completely removed from the endotracheal tube. This method can be selected for patients who are expected to have difficulty in nasotracheal intubation, but it is not recommended as it is time-consuming and may require complicated maneuvers [52-54].

\section{Airway care after nasotracheal intubation}

After endotracheal intubation, the stable depth was checked and fixed. In most cases, the intubation depth is calculated based on orotracheal intubation, while a deeper depth is selected for nasotracheal intubation. Improper depth of endotracheal intubation may cause endobronchial intubation or damage to the vocal cords or bronchial tubes by the cuff or tube tip. The exact position of the endotracheal tube tip is the mid-trachea when the head of the patient is in a neutral position. It is selected considering the correlation with height, the sum of the distances from nares to the tragus, tragus to the angle of the mandible, and angle of the mandible to the sternal notch $[55,56]$.

Several methods can be used to confirm the correct placement of the endotracheal tube. Usually, it is confirmed by auscultation of breathing sounds, but X-ray and fiberoptic bronchoscopy are applied as gold standards for confirmation of correct placement. In general, the mean distance from nares to the tip of endotracheal tube tip for nasotracheal intubation is $28.9 \pm 1.3 \mathrm{~cm}$ in men and $26.6 \pm 1.5 \mathrm{~cm}$ in women. In addition, palpation of the tube cuff in the suprasternal notch is an easy, fast, and cheap method $[57,58]$.

In general, the exact position of the tube is $2-3 \mathrm{~cm}$ above the carina [48]. In addition to X-ray and fiberoptic bronchoscopy, ultrasonography can be used as a reliable method. Ultrasonography has the advantages of good mobility, safety, and good correlation compared to X-rays [55,59]. In adults, the length of endotracheal tube insertion varies depending on height. There is a method of presenting based on weight, but it is important to check the stable depth after insertion [60].

After endotracheal intubation, the depth of the tube was checked and fixed for stable management under anesthesia without interfering with the surgery. It may be possible to simply use adhesive tape or apply auxiliary devices while preventing the patient from being injured by pressure caused by fixing the tube. Recently, there has been a method of making it suitable for patients using a 3D printer [61].

\section{Nasotracheal intubation in pediatric patients}

In pediatric patients, there is also a method for determining the length of the endotracheal tube based on the internal diameter of the endotracheal tube. In other words, it is a method of measuring length by multiplying the tube size by three, but it is not an accurate method, resulting in malposition in $15-25 \%$ of cases [62]. In addition, the cuffed pediatric endotracheal tube (Microcuff ${ }^{\circledast}$ Pediatric Tracheal Tube, MPTT, Microcuff GmBH, Germany) used in children has a high volume with a low-pressure cuff. It is characterized by a small-sized cuff, low sealing cuff pressure, and a polyurethane tube with no Murphy's eye. Confirmation of the endotracheal tube length using the depth marking of this tube allows safe mounting without risk of endobronchial intubation [63]. However, it should be noted that the black line may not be a depth marker depending on the tube manufacturer [64]. In children, the depth of the tube also changes depending on the head position. In other words, it should be remembered that the tube moves toward the carina during 
flexion, away from the carina during extension and lateral rotation, resulting in malposition of the tube. It is important to maintain a neutral position, that is, a sniffing position [65,66].

Using a tube with a cuff requires attention to the cuff pressure. When the cuff pressure reaches $30 \mathrm{cmH}_{2} \mathrm{O}$, the tracheal mucosal blood flow is compromised, and when the cuff pressure reaches $45 \mathrm{cmH}_{2} \mathrm{O}$, the mucosal blood flow is completely obstructed [21]. It has been reported that tube lubrication with K-Y jelly (Johnson \& Johnson, USA) inhibits the increase in cuff pressure in vitro when using a tube with a cuff under general anesthesia using $\mathrm{N}_{2} \mathrm{O}$. However, its clinical significance is limited [67]. The advantage of using an uncuffed tube in children is that a tube with a larger internal diameter can be used, thereby reducing airway resistance and work of breathing. However, leakage may occur because of incomplete sealing. In this case, leakage can be reduced by throat pack placement and mouth opening. The reason for the decrease in leakage when the mouth is open is that the mandible moves toward the larynx to decrease the thyromental distance, thereby physically compressing the soft tissue in between. This method improves sealing and reduces leakage [68]. Adequate endotracheal tube length can be predicted by using the equations for predicting the adequate tube depths in Japanese patients: (patient's height / 10) - 3 + DM (distance between the distal edge of intubation guide mark and the tip, in $\mathrm{cm}$ ) for oral intubation, and (patient's height / 10) + 1 + DM for nasal intubation $[19,69]$.

\section{Instruments for nasotracheal intubation}

\section{Endotracheal tube}

1) Tube type

(1) Standard endotracheal tube: This refers to the use of a conventional tracheal tube for orotracheal intubation. Standard endotracheal tubes that can be used for nasotracheal intubation can be classified into polyvinyl chloride, rubber, and polyurethane tubes, depending on the material, and armored tubes that emphasize flexibility. In nasotracheal intubation, care must be taken as the tube is hard and likely to damage the surrounding tissues, causing complications such as epistaxis. In some cases, the end of the tracheal tube is warmed to soften the end to reduce damage during insertion $[70,71]$. In addition, if the length of the tube is short and is inserted at an appropriate depth, the connection between the en- dotracheal tube and the breathing circuit is located at the entrance of the nasal cavity. Care should be taken to avoid damage to the nares and disconnection, affecting not only ventilation but also the surgical field of view. Unlike orotracheal intubation, a tube with a small diameter is chosen to pass through the nasal cavity, but it should not be excessively small [2-4]. Tubes without cuffs can be inserted smoothly, but to prevent air leakage, they are packed in the peri-cuff area for clinical application. In general, a nasal tube with a longer length and smaller diameter tends to be selected, thereby increasing the resistance to breathing [3-5].

(2) RAE (Ring, Adair, Elwyn) endotracheal tube: The endotracheal tube for nasotracheal intubation is longer than that for orotracheal intubation and is bent upward or downward at the nasal entrance so that it can be fixed without obstructing the surgical field of view. It is more effective because it is longer and more flexible than a standard endotracheal tube.

(3) Parker Flex-tip nasal endotracheal tube: Owing to the left-sided bevel and right tip orientation of a conventional tube, insertion through the right nostril during nasal passage is likely to cause turbinate damage, while insertion through the left nostril is likely to cause damage to the septum. On the other hand, a Parker Flex-tip tube is composed of a posterior bevel and an anterior tip so that the tip and bevel are not in contact with the turbinate and septum. In addition, the soft and flex tips are in contact with a wider area and pass smoothly along the curve of the nasopharynx. Therefore, it results in less nasal mucosal trauma and epistaxis and is suitable for nasotracheal intubation [72,73]. However, some argue that the softness of the tube tip is more important than the shape of the tube. In addition, the surgical procedure may be difficult because of vertical protrusion from the nostril. Another disadvantage is that the risk of airway obstruction may increase because of the high chance of tube kinking (Fig. 1) [73,74].

\section{2) Shape of the endotracheal tube tip}

A conventional tube with a Murphy eye is called a Murphy-tip, and a sharp-edged Murphy eye has a high probability of causing mucosal trauma in the process of passing through the nasal cavity. A Magill tip has no Murphy eye and has a shorter and blunter tip, which is less likely to cause nasal mucosal trauma [75]. 


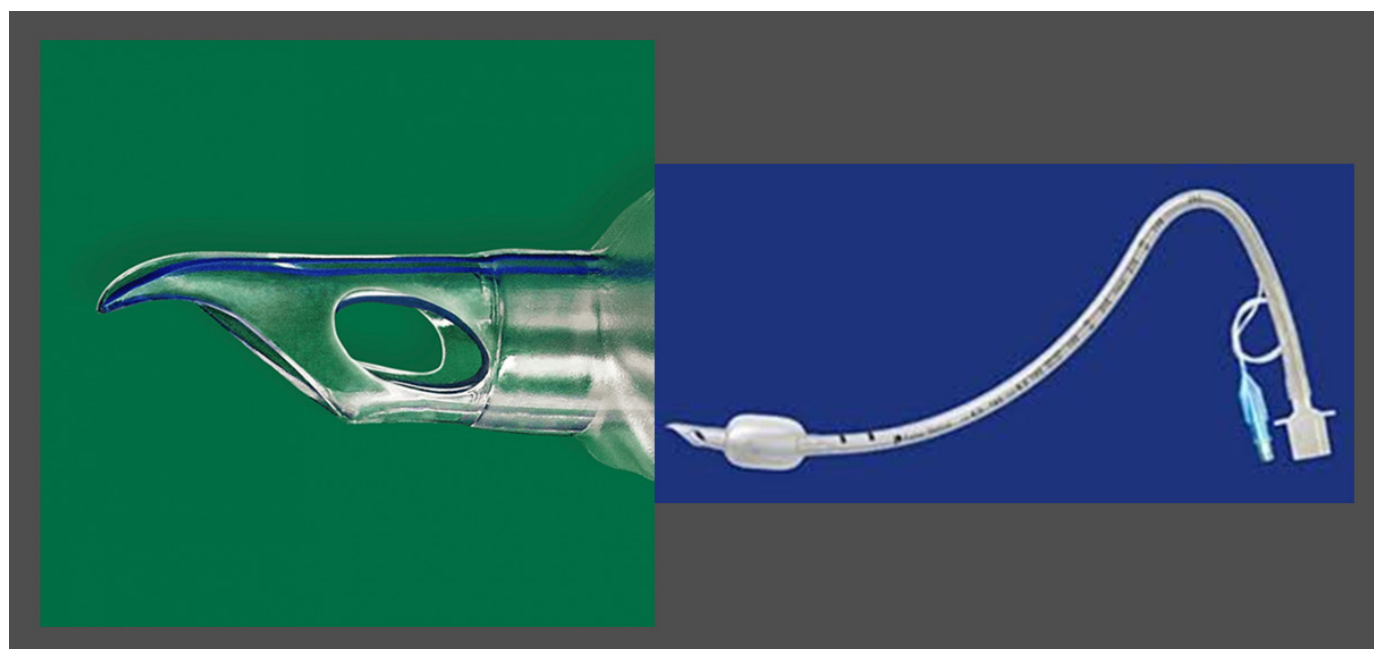

Fig. 1. Parker Flex-tip nasal endotracheal tube.

\section{Laryngoscope}

The laryngoscope is an instrument that can directly visualize and identify the structures constituting the upper airway, which plays an important role in endotracheal intubation. A direct laryngoscope serves as the basic standard technique for endotracheal intubation. It guides the path for the endotracheal tube as it passes through the vocal cord and can be easily inserted into the airway by exposing the inside of the larynx during the process of securing the airway. Another method is to attempt endotracheal intubation indirectly. Some methods are gradually disappearing, while there are methods newly applied to clinical practice, such as methods using malleable or rigid optical stylets and methods using a rigid indirect laryngoscope (e.g., Bullard laryngoscope, TruView $\mathrm{EVO}_{2}$, fiberoptic, and video-laryngoscope) [75].

\section{1) Direct laryngoscope and blade}

Recently, blades used with direct laryngoscopes have been supplied in various shapes and materials, including disposable and reusable ones, and it is possible to choose a blade of the size and length that suits the patient's condition.

(1) Macintosh blade: The Macintosh blade provides a view of the larynx by mounting the tip of the blade on the vallecula and indirectly lifting the epiglottis. The base of the epiglottis is suspended in the hyoepiglottic ligament and hyoid bone. When the hyoepiglottic ligament is lifted in the distal direction, the epiglottis is elevated to visualize the glottic opening. The large flange of the Macintosh blade is advantageous for the displacement of the tongue, and the curved blade lifts the epiglottis, facilitating intuba- tion [76].

(2) Miller blade: The Miller blade was placed under the epiglottis to visualize the laryngeal structures. Since it is a blade that exposes the glottic opening, it is useful for a large, floppy, and irregularly shaped epiglottis. The small spatula and flange make it particularly useful for patients with smaller displacement spaces. In other words, it is useful in children and patients with micrognathia, prominent upper incisors, and short mental-hyoid distance. Therefore, it provides a better view of the laryngeal structures [76].

(3) McCoy blade: The McCoy blade was developed in the early 1990s for difficult intubation in adult patients. It has an articulating distal tip to improve the lift of the epiglottis $[76,77]$.

\section{2) Indirect laryngoscope}

Indirect laryngoscopes include optical stylets, rigid fiberoptic laryngoscopes, rigid video-laryngoscopes, and flexible fiberoptic laryngoscopes.

(1) Optical stylets: Optical stylets included the Shikani Seeing Stylet (Clarus Medical LLC, USA), Fiberlightview Shuttle (Anesthesia Medical Specialties, USA), video optical intubation stylet (Velpi AG, Switzerland), and Bonfils intubation fiberscope (Karl Storz Endoscopy, Germany). Compared to the malleable Shikani Seeing Stylet, Bonfils is a stylet modified by applying a fixed curve to the distal end of the initial rigid and straight design. Both types have good controllability and can be used to open the mouth enough to fit the endotracheal tube [76,77]

(2) Rigid fiberoptic laryngoscope: It includes the Bullard la- 
ryngoscope, WuScope, UpsherScope, and TruView $\mathrm{EVO}_{2}$. The Bullard laryngoscope, introduced in 1988, enables intubation even in patients with limited mouth opening with a dedicated intubating stylet [76]. TruView $\mathrm{EVO}_{2}$ facilitates easy observation and operation of the laryngoscope with a digital camera mounted on the viewport. Rigid-type laryngoscopes are not widely used because they are not flexible and are less popular than video-laryngoscopes [76].

(3) Rigid video-laryngoscopy: It includes Airtraq, GlideScope, Pentax Airway Scope, McGrath, CoPilot, and Storz C-MAC. The video-laryngoscope resembles a direct laryngoscope. A video chip was attached to show the laryngeal view. It shows the larynx better than the direct laryngoscope and is the first choice when difficult intubation is expected. However, it should be noted that providing a good laryngeal view does not always facilitate intubation [76-78]. The C-MAC has a blade similar to the shape of a Macintosh laryngoscope and requires a technique similar to that of a direct laryngoscope. The C-MAC video-laryngoscope is almost always used for difficult airway management. Alternatively, GlideScope, McGrath, and CoPilot have an angulated blade with a sharper curve [79]. The GlideScope has a high-resolution video camera on the laryngoscope blade, which varies in size from child to adult. The McGrath can be used by inserting a single-use blade with a view monitor placed on the handle with a disposable battery. The monitor can be tilted and swiveled to adjust the position of the view $[77,80]$. The CoPilot has a C-shaped bougie port channel on the laryngoscope blade, and the endotracheal tube passes through the bougie, making it easy to mount the tube. This videolaryngoscope is excellent for teaching, as educators and trainees can share views at the same time. The view is limited in direct laryngoscopy because the distance between the glottis and the viewer only provides a view angle of $15-30^{\circ}$. The camera lens is near the tip of the laryngoscope with a view angle of $50-60^{\circ}$ in the videolaryngoscope, allowing a better laryngeal view [76]. Lastly, Airtraq cannot be used because it has a reusable optic piece and is used in intubation by loading an endotracheal tube through a guide channel; however, the use of Airtraq (Airtraq NT) for nasotracheal intubation is considered possible [81].

\section{Lightwand}

In oral or maxillofacial surgery where a difficult airway is expected, the lightwand is an easy-to-use and economical device when a fiberoptic laryngoscope cannot be used. It has the advantage of a high success rate and is hemodynamically stable, causing fewer complications. The advantage of the new version, the lightwand, Trachlight, is that it is easy to use, has a bright light source, can be projected anteriorly and laterally, and can be used for both oral and nasal intubation. However in obese patients, it is difficult to observe external light. In addition, its use is limited as it is contraindicated in tumors, infections, trauma, and foreign body cases $[50,82,83]$.

\section{Magill forceps}

After insertion of the endotracheal tube through the nasal cavity, the provider safely holds the distal part of the endotracheal tube with the Magill forceps. The endotracheal tube is inserted into the larynx under the laryngoscope during nasotracheal intubation without trauma to the patient and without damaging the tube or cuff. Sufficient length is required with a good handle of the tube, and the handle should not interfere with the view, occupy minimal space, and should be held firmly [84].

There are several modified Magill forceps. The Boedeker forceps, which are curved intubating forceps, are designed to facilitate the removal of foreign bodies with the distal forceps well seen on the videolaryngoscope. The forceps are bent at two points for easy handling of the tube in the glottis, and the modified Magill forceps facilitate intubation into the cephalad larynx and the posterior angled trachea in children [85-87].

\section{Endotracheal tube introducer (bougie)}

This is not a common choice, but it may be chosen according to the preferences and skills of the anesthesiologist and the patient's condition. In general, there should be at least two introducers for nasotracheal intubation. One introducer was inserted through the nasal cavity to be connected to the oropharynx, with another introducer inserted through the oral cavity to the bronchus. After nasotracheal intubation, both introducers can be removed through the endotracheal tube, but they must be flexible enough to reduce damage; therefore, material selection is important $[88,89]$.

\section{Indications and contraindications for nasotracheal intubation}

\section{Indications for nasotracheal intubation}

In oral and maxillofacial surgeries, such as intraoral or 
mandibular surgeries, nasotracheal intubation has been chosen as the route of choice because it ensures a stable airway without disturbing the surgical field. It is a useful technique when orotracheal intubation is difficult, for example, trismus, previous radiation therapy, and oropharyngeal infection (Table 1) [3-9].

\section{Contraindications for nasotracheal intubation}

Nasotracheal intubation may result in complications or an increased risk of injury, such as penetrating trauma of the frontal lobe through the cribriform plate as the endotracheal tube is inserted due to basilar skull fracture during nasotracheal intubation [2-5]. In patients with clotting factor deficiency, severe epistaxis may be caused by mucosal trauma during tube progression through the nasal cavity [2-6]. Patients should be thoroughly checked for antiplatelet medication, anticoagulation medication, or hereditary telangiectasia. Care should be taken in patients with cardiac valvular abnormalities or prosthetic valves, as there is a high risk of tube-induced bacteremia. In addition, blind nasotracheal intubation may be contraindicated in patients with upper airway foreign bodies or severe facial trauma (Table 2) [2-5,44].

\section{Complications}

\section{Types of complications}

1) Epistaxis: Epistaxis is the most common complication of nasal mucosal trauma. It is mainly caused by damage to Kisselbach's plexus in Little's area, located in the anterior region of the nasal septum. This may be caused by the large size of the endotracheal tube, excessive force, repeated attempts, and inappropriate vasoconstriction. During severe epistaxis, there is a possibility of pulmonary aspiration, and it is necessary to control bleeding by packing the posterior nasal space [5-7].

2) Bacteremia: When performing the procedure around the oral cavity under general anesthesia, the incidence of bac-

Table 1. Indications of Nasotracheal Intubation

Intranasal and oropharyngeal surgery
Mandible surgery
Maxillofacial surgery
Limited mouth opening due to trismus
Dental surgery
Rigid laryngoscopy and microlaryngeal surgery
Cervical spine instability or severe degenerative cervical spine dis-
ease

teremia is high. This may be caused by the invasion of the nasal flora into the highly vascularized trachea. Thus, prophylactic antibiotic administration should be considered in patients with prosthetic cardiac valves. The causative bacteria include alpha-hemolytic Streptococcus and Corynebacterium species [2-4].

3) Obstruction of the nasotracheal tube: Obstruction may occur due to avulsion of the nasal polyp or inferior turbinate, tooth, and blood clot; obstruction of the main bronchus may cause serious ventilatory disturbance [2-6].

4) Retropharyngeal perforation: Laceration is caused by dissection of the retropharyngeal mucosa, which may lead to perforation in severe cases. If resistance occurs in the tube passage or if a breathing sound is not heard, it should be recognized that the tube is already located on the pharyngeal wall. If there is a possibility of complications, broad-spectrum antibiotics should be administered to reduce the risk of infection [4-6].

5) Perforation of pyriform fossa: Pyriform fossa perforation can lead to subcutaneous and mediastinal emphysema. Therefore, care should be taken to monitor the condition of critically ill patients [2-4].

6) Sinusitis: Factors that can cause sinusitis include the presence of foreign bodies in the nasal cavity, retention of secretions, bacterial colonization, immunosuppression, supine position, and variation in the size of sinus ostium. It can be diagnosed using sinus needle aspiration or culture [4-6].

7) Pneumonia: Pneumonia can be caused by various factors, and its prevalence is higher in patients who undergo nasotracheal intubation than in those who undergo orotracheal intubation [3-5].

8) Sepsis: Pneumonia, sinusitis, and sepsis are the most frequent late complications. Sepsis can be diagnosed based on positive findings and clinical evidence of infection in

Table 2. Contraindications of Nasotracheal Intubation

Basilar skull fractures combined with or without cerebrospinal fluid
leakage
Coagulopathy
Epiglottitis
Nasal foreign bodies
Nasal polyp
Frequent episodes of epistaxis
Prosthetic heart valves
Nasal bone fracture
Patients with facial trauma such as facial bone fracture


blood cultures [3-5].

9) Other complications: Inadvertent intracranial placement of the nasotracheal tube can cause fracture of the cribriform plate due to damage to the middle turbinate, anosmia due to olfactory nerve injury, CSF rhinorrhea, meningitis, hemiparesis, and blindness due to brain damage in severe cases [2-7]. It may also result in inferior turbinate ulceration, laryngitis, vocal cord paralysis, otitis media due to obstruction of the Eustachian tube by a nasotracheal tube, and necrosis of the nasal ala. With repeated insertion into the esophagus, especially in the presence of a malignancy or inflammation in the esophageal wall, esophageal perforation may be induced (Table 3) [2-6,44].

\section{Risk factors for complications}

In the preoperative examination, the medical history and patient's conditions related to risk factors that may cause complications from nasotracheal intubation should be checked (Table 4) [2-6].

\section{Prevention}

The methods that can be applied to prevent complications caused by nasotracheal intubation are listed below.

1. After nasotracheal intubation, several methods have been used to prevent and reduce the risk of epistaxis, which is the most common complication. One of the methods is to use topical vasoconstrictors such as epinephrine, phenylephrine, xylometazoline, and oxymetazoline. However, as these may cause life-threatening complications such as

Table 3. Complications of Nasotracheal Intubation

Epistaxis
Bacteremia
Obstruction of the nasotracheal tube
Retropharyngeal perforation
Perforation of pyriform fossa
Sinusitis
Pneumonia
Sepsis
Other complications
CSF rhinorrhea due to the intracranial placement of the naso-
tracheal tube, brain damage, meningitis, hemiparesis, blind-
ness, fracture of the cribriform plate, olfactory nerve injury and
anosmia, inferior turbinate ulceration, laryngitis, vocal cord pa-
ralysis, otitis media caused by Eustachian tube obstruction,
necrosis of nasal ala, esophagus intubation, esophageal per-
foration

CSF: cerebrospinal fluid. arrhythmia, myocardial infarction, and cardiac arrest, care should be taken when choosing the drug and dosage [90].

2. It has been reported that the same preventive effect for epistaxis as a thermosoftened tube could be obtained by inserting an esophageal stethoscope into the endotracheal tube, ballooning the stethoscope, and obstructing the tip of the tube with the tip of the stethoscope [90]. Similarly, it has been reported that the combination of tube softening and red-rubber catheter-guided intubation reduces epistaxis $[91,92]$.

3. Nasotracheal intubation under a curve-tipped suction catheter guide and intubation using a "Bubble-tip" (Airguide $^{\circledast}$ ) tracheal tube system are also introduced as methods to reduce epistaxis [91-93].

4. Since the Parker Flex-Tip tube has a posteriorly oriented bevel and slides along the posterior wall, it has been reported to reduce the epithelial stripping of the turbinate and septum, and the soft flex tip contacts the mucosa on many surfaces to reduce tissue pressure [72,73].

5. Because lower pathway intubation of the nose decreases the trauma and epistaxis of the middle turbinate, tube advancement between the floor of the nose and inferior turbinate is a better approach for the prevention of trauma [2-9].

6. Reinforced tubes are slightly larger in external diameter than relatively firm and inflexible preformed tubes, but their flexibility allows them to easily enter narrow pathways, resulting in easier tube passage through the lower pathway $[8,9]$.

7. The Murphy tip endotracheal tube can induce trauma of the nasal mucosa as the sharp-edged Murphy eye passes through the nasal cavity. However, if there is no murphy eye, the shorter Magill-tip endotracheal tube has a blunt tip, causing a small impaction of the nasal mucosa, thereby reducing trauma [75].

Table 4. Risk Factors for Complications

Abnormal anatomy: septal deviation, etc.
Pre-existing medical condition:
Gastroesophageal reflux disorder,
Coagulopathy or antiplatelet medication
Large tube size selection
Cuff pressure too high
Presence of nasogastric tube
Excessive force during intubation
Repeated tube insertion
Swelling or infection of the surrounding organs, such as sinusitis


8. The use of a silicone tube with a soft and round tip, although expensive, can reduce epistaxis or nasal complications. Methods to prevent and reduce the complications of nasotracheal intubation include the use of mechanical dilation, sterile lubrication, water-soluble jelly, tubes with smaller diameters, gentle tubes, and softened tube tips by warming up $[29,75,90]$.

9. In patients with head and neck tumors, it may be difficult to maintain and manage airways due to anatomical and physiological changes, as well as radiation therapy and chemotherapy [22]. It should be approached in consideration of the fact that tumors may cause difficulties in airway management due to abnormalities such as receding jaw, restricted mouth opening, and neck movement abnormalities. Depending on the size and location of the tumor, complications such as obstruction and bleeding in the nasal cavity, through which the tracheal tube passes, and the selection and placement of the laryngoscope and blade may affect the surgical field of vision [94,95].

10. The restriction of neck movement, especially neck stiffness, is often accompanied by intubation difficulties due to insufficient neck extension due to contracture caused by radiation therapy. Adequate preoperative judgment, mask ventilation, and endotracheal intubation during surgery should be prepared to prevent complications and to reduce airway-related morbidity, along with a reliable plan to ensure safe airway management [94,95].

\section{CONCLUSION}

Nasotracheal intubation is a basic procedure that replaces orotracheal intubation in oral or maxillofacial surgery and intensive care management. By learning and acquiring techniques for direct laryngoscopy, video-laryngoscopy, use of Magill forceps, and selection of an endotracheal tube for nasotracheal intubation, which is being applied as a basic method for all airway management from blind nasotracheal intubation, it will be possible to safely manage the airway. This will also allow quick coping with unexpected difficulties in intubation, securing the view of an appropriate surgical field, and preventing side effects and complications from the insertion process.

\section{CONFLICTS OF INTEREST}

No potential conflict of interest relevant to this article was reported.

\section{DATA AVAILABILITY STATEMENT}

Not applicable.

\section{AUTHOR CONTRIBUTIONS}

Conceptualization: Dong Ho Park, Chia An Lee, Chang Young Jeong, Hong-Seuk Yang. Writing - original draft: Dong Ho Park, Chia An Lee. Writing - review \& editing: Dong Ho Park, Chia An Lee, Hong-Seuk Yang. Supervision: Chang Young Jeong, Hong-Seuk Yang.

\section{ORCID}

Dong Ho Park, https://orcid.org/0000-0002-6587-3756

Chia An Lee, https://orcid.org/0000-0001-9143-7133

Chang Young Jeong, https://orcid.org/0000-0002-8830-3406

Hong-seuk Yang, https://orcid.org/0000-0003-2023-8705

\section{REFERENCES}

1. Duncan JA. Intubation of the trachea in the conscious patient. Br J Anaesth 1977; 49: 619-23.

2. Dauphinee K. Nasotracheal intubation. Emerg Med Clin North Am 1988; 6: 715-23.

3. Prasanna D, Bhat S. Nasotracheal intubation: an overview. J Maxillofac Oral Surg 2014; 13: 366-72.

4. Holzapfel L. Nasal vs oral intubation. Minerva Anestesiol 2003; 69: $348-52$

5. Hall CE, Shutt LE. Nasotracheal intubation for head and neck surgery. Anaesthesia 2003; 58: 249-56.

6. Tintinalli JE, Claffey J. Complications of nasotracheal intubation. Ann Emerg Med 1981; 10: 142-4.

7. Roppolo LP, Vilke GM, Chan TC, Krishel S, Hayden SR, Rosen P, et al. Nasotracheal intubation in the emergency department, revisited. J Emerg Med 1999; 17: 791-9.

8. Sun Y, Jiang H, Zhu Y, Xu H, Huang Y. Blind intubation device for nasotracheal intubation in 100 oral and maxillofacial surgery patients with anticipated difficult airways: a prospective evaluation. Eur J Anaesthesiol 2009; 26: 746-51.

9. Chauhan V, Acharya G. Nasal intubation: a comprehensive review. Indian J Crit Care Med 2016; 20: 662-7.

10. Luckhaupt H, Brusis T. [History of intubation]. Laryngol Rhinol Otol (Stuttg) 1986 65: 506-10. German.

11. Robinson DH, Toledo AH. Historical development of modern anesthesia. J Invest Surg 2012; 25: 141-9.

12. Magill IW. Technique in endotracheal anaesthesia. Br Med J 
1930; 2: 817-9.

13. Magill IW. Blind nasal intubation. Anaesthesia 1975; 30: 476-9.

14. Rowbotham S. Intratracheal anaesthesia by the nasal route for operations on the mouth and lips. Br Med J 1920; 2: 590-1.

15. O'Reilly MJ, Reddick EJ, Black W, Carter PL, Erhardt J, Fill W, et al. Sepsis from sinusitis in nasotracheally intubated patients. A diagnostic dilemma. Am J Surg 1984; 147: 601-4.

16. Raj PP, Forestner J, Watson TD, Morris RE, Jenkins MT. Technics for fiberoptic laryngoscopy in anesthesia. Anesth Analg 1974; 53: 708-14.

17. Arrowsmith JE, Robertshaw HJ, Boyd JD. Nasotracheal intubation in the presence of frontobasal skull fracture. Can J Anaesth 1998; 45: 71-5.

18. Teul I, Slawinski G, Lewandowski J, Dzieciolowska-Baran E, Gawlikowska-Sroka A, Czerwinski F. Nasal septum morphology in human fetuses in computed tomography images. Eur J Med Res 2010; 15(Suppl 2): 202-5.

19. Kemper M, Dullenkopf A, Schmidt AR, Gerber A, Weiss M. Nasotracheal intubation depth in paediatric patients. Br J Anaesth 2014; 113: 840-6.

20. Santillanes G, Gausche-Hill M. Pediatric airway management. Emerg Med Clin North Am 2008; 26: 961-75.

21. Shohara K, Goto T, Kuwahara G, Isakari Y, Moriya T, Yamamuro T. Validity of rhinometry in measuring nasal patency for nasotracheal intubtion. J Anesth 2017; 31: 1-4.

22. Pearce A. Evaluation of the airway and preparation for difficulty. Best Pract Res Clin Anaesthesiol 2005; 19: 559-79.

23. Prasad A, Yu E, Wong DT, Karkhanis R, Gullane P, Chan VW. Comparison of sonography and computed tomography as imaging tools for assessment of airway structures. J Ultrasound Med 2011; 30: 965-72.

24. Chi SI, Park S, Joo LA, Shin TJ, Kim HJ, Seo KS. Identifying the more suitable nostril for nasotracheal intubation using radiographs. J Dent Anesth Pain Med 2016; 16: 103-9.

25. Rajan S, Arora V, Tosh P, Mohan P, Kumar L. Effectiveness of transtracheal lidocaine as an adjunct to general anesthesia in providing patient immobility during total parotidectomy: a comparison with dexmedetomidine infusion. J Anaesthesiol Clin Pharmacol 2017; 33: 193-6.

26. Gold MI, Buechel DR. Translaryngeal anesthesia: a review. Anesthesiology 1959; 20: 181-5.

27. Baraka AS, Taha SK, Aouad MT, El-Khatib MF, Kawkabani NI. Preoxygenation: comparison of maximal breathing and tidal volume breathing techniques. Anesthesiology 1999; 91: 612-6.

28. Nimmagadda U, Salem MR, Crystal GJ. Preoxygenation: physiologic basis, benefits, and potential risks. Anesth Analg 2017; 124: 507-17.
29. Tan P, Dennis AT. High flow humidified nasal oxygen in pregnant women. Anaesth Intensive Care 2018; 46: 36-41.

30. Patel A, Nouraei SA. Transnasal humidified rapid-insufflation ventilatory exchange (THRIVE): a physiological method of increasing apnoea time in patients with difficult airways. Anaesthesia 2015; 70: 323-9.

31. Pillai A, Daga V, Lewis J, Mahmoud M, Mushambi M, Bogod D. High-flow humidified nasal oxygenation vs. standard face mask oxygenation. Anaesthesia 2016; 71: 1280-3.

32. Humphreys S, Lee-Archer P, Reyne G, Long D, Williams T, Schibler A. Transnasal humidified rapid-insufflation ventilatory exchange (THRIVE) in children: a randomized controlled trial. Br J Anaesth 2017; 118: 232-8.

33. Andel H, Klune G, Andel D, Felfernig M, Donner A, Schramm W, et al. Propofol without muscle relaxants for conventional or fiberoptic nasotracheal intubation: a dose-finding study. Anesth Analg 2000; 91: 458-61.

34. Ide M, Sunada K, Katsuyama N. Are muscle relaxants needed for nasal intubation in propofol and remifentanil anesthesia? J Oral Maxillofac Surg 2014; 72: 2134-9.

35. Tramèr MR, Fuchs-Buder T. Omitting antagonism of neuromuscular block: effect on postoperative nausea and vomiting and risk of residual paralysis. A systematic review. Br J Anaesth 1999; 82: 379-86.

36. Kihara S, Komatsuzaki T, Brimacombe JR, Yaguchi Y, Taguchi N, Watanabe S. A silicone-based wire-reinforced tracheal tube with a hemispherical bevel reduces nasal morbidity for nasotracheal intubation. Anesth Analg 2003; 97: 1488-91.

37. Kim H, Lee JM, Lee J, Hwang JY, Chang JE, No HJ, et al. Effect of neck extension on the advancement of tracheal tubes from the nasal cavity to the oropharynx in nasotracheal intubation: a randomized controlled trial. BMC Anesthesiol 2019; 19: 158.

38. Horton WA, Fahy L, Charters P. Defining a standard intubating position using "angle finder". Br J Anaesth 1989; 62: 6-12.

39. Vivian V, van Zundert AA. Nasotracheal intubation and epistaxis. Anaesthesia 2016; 71: 722-3.

40. Zhang J, Lamb A, Hung O, Hung C, Hung D. Blind nasal intubation: teaching a dying art. Can J Anaesth 2014; 61: 1055-6.

41. Dubey PK, Dubey P, Kumar N, Bhardwaj G, Kumar N. Blind nasal intubation revisited: no longer a blind technique? J Emerg Med 2017; 52: 231-4.

42. Jones PM, Armstrong KP, Armstrong PM, Cherry RA, Harle CC, Hoogstra J, et al. A comparison of glidescope videolaryngoscopy to direct laryngoscopy for nasotracheal intubation. Anesth Analg 2008; 107: 144-8.

43. Puchner W, Drabauer L, Kern K, Mayer C, Bierbaumer J, Rehak $\mathrm{PH}$, et al. Indirect versus direct laryngoscopy for routine naso- 
tracheal intubation. J Clin Anesth 2011; 23: 280-5.

44. Lim D, Ma BC, Parumo R, Shanmuhasuntharam P. Thirty years of submental intubation: a review. Int J Oral Maxillofac Surg 2018; 47: 1161-5.

45. Kwon MA, Song J, Kim S, Ji SM, Bae J. Inspection of the nasopharynx prior to fiberoptic-guided nasotracheal intubation reduces the risk epistaxis. J Clin Anesth 2016; 32: 7-11.

46. Park YH, Choi YJ, Choi WC, Lee UL. Partially withdrawn nasotracheal tube: an alternative to the nasal trumpet. Anesth Prog 2015; 62: 166-7.

47. Halligan M, Charters P. A clinical evaluation of the Bonfils intubation fibrescope. Anaesthesia 2003; 58: 1087-91.

48. Byhahn C, Nemetz S, Breitkreutz R, Zwissler B, Kaufmann M, Meininger D. Brief report: tracheal intubation using the Bonfils intubation fibrescope or direct laryngoscopy for patients with a simulated difficult airway. Can J Anaesth 2008; 55: 232-7.

49. Iseki K, Murakawa M, Tase C, Otsuki M. Use of a modified lightwand for nasal intubation. Anesthesiology 1999; 90: 635.

50. Dong Y, Li G, Wu W, Su R, Shao Y. Lightwand-guided nasotracheal intubation in oromaxillofacial surgery patients with anticipated difficult airways: a comparison with blind nasal intubation. Int J Oral Maxillofac Surg 2013; 42: 1049-53.

51. Abrons RO, Vansickle RA, Ouanes JP. Seldinger technique for nasal intubation: a case series. J Clin Anesth 2016; 34: 609-11.

52. Mahajan R, Ahmed P, Shafi F, Bassi R. Dual bougie technique for nasotracheal intubation. Anesth Prog 2012; 59: 85-6.

53. Arisaka H, Sakuraba S, Furuya M, Higuchi K, Yui H, Kiyama S, et al. Application of gum elastic bougie to nasal intubation. Anesth Prog 2010; 57: 112-3.

54. Cooper RM. Conversion of a nasal to an orotracheal intubation using an endotracheal tube exchanger. Anesthesiology 1997; 87: 717-8.

55. McKay WP, Klonarakis J, Pelivanov V, O’Brien JM, Plewes C. Tracheal palpation to assess endotracheal tube depth: an exploratory study. Can J Anaesth 2014; 61: 229-34.

56. Ji SM. Estimation of optimal nasotracheal tube depth in adult patients. J Dent Anesth Pain Med 2017; 17: 307-12.

57. Okuyama M, Imai M, Sugawara K, Okuyama A, Kemmotsu O. [Finding appropriate tube position by the cuff palpation method in children]. Masui 1995 44: 845-8. Japanese.

58. Pollard RJ, Lobato EB. Endotracheal tube location verified reliably by cuff palpation. Anesth Analg 1995; 81: 135-8.

59. Dennington D, Vali P, Finer NN, Kim JH. Ultrasound confirmation of endotracheal tube position in neonates. Neonatology 2012; 102: 185-9.

60. Massoth C, Schülke C, Köppe J, Weiss R, Pöpping D, Dahrmann $\mathrm{M}$, et al. Nasolaryngeal distances in the adult population and an evaluation of commercially available nasotracheal tubes. Anesth Analg 2020; 130: 1018-25.

61. Koshika K, Hoshino T, Shibata Y, Ouchi T, Koitabashi T. New device for securing nasotracheal intubation tube during oral and maxillofacial surgery. Bull Tokyo Dent Coll 2020; 61: 275-9.

62. Phipps LM, Thomas NJ, Gilmore RK, Raymond JA, Bittner TR, Orr RA, et al. Prospective assessment of guidelines for determining appropriate depth of endotracheal tube placement in children. Pediatr Crit Care Med 2005; 6: 519-22.

63. Weiss M, Balmer C, Dullenkopf A, Knirsch W, Gerber ACh, Bauersfeld U, et al. Intubation depth markings allow an improved positioning of endotracheal tubes in children. Can J Anaesth 2005; 52: 721-6.

64. Goel S, Lim SL. The intubation depth marker: the confusion of the black line. Paediatr Anaesth 2003; 13: 579-83.

65. Conrardy PA, Goodman LR, Lainge F, Singer MM. Alteration of endotracheal tube position. Flexion and extension of the neck. Crit Care Med 1976; 4: 8-12.

66. Donn SM, Kuhns LR. Mechanism of endotracheal tube movement with change of head position in the neonate. Pediatr Radiol 1980; 9: 37-40.

67. Oji M, Koyama Y, Oshika H, Kohno M, Nakahashi Y, Fukushima S, et al. Effect of endotracheal tube lubrication on cuff pressure increase during nitrous oxide exposure: a laboratory and prospective randomized controlled trial. BMC Anesthesiol 2019; 19: 169.

68. Bradford KE, Siegelman LI, Psoter WJ. The effects of mouth opening and throat pack placement on uncuffed nasal endotracheal tube leakage and delivered tidal volumes in mechanically ventilated pediatric patients undergoing dental procedures. Anesth Prog 2018; 65: 237-43.

69. Yao K, Goto K, Nishimura A, Shimazu R, Tachikawa S, Iijima T. A formula for estimating the appropriate tube depth for intubation. Anesth Prog 2019; 66: 8-13.

70. Hosseinzadeh H, Taheri Talesh K, Golzari SE, Gholizadeh H, Lotfi A, Hosseinzadeh P. Warming endotracheal tube in blind nasotracheal intubation throughout maxillofacial surgeries. J Cardiovasc Thorac Res 2013; 5: 147-51.

71. Kim EM, Chung MH, Lee MH, Choi EM, Jun IJ, Yun TH, et al. Is tube thermosoftening helpful for videolaryngoscope-guided nasotracheal intubation?: a randomized controlled trial. Anesth Analg 2019; 129: 812-8.

72. Sanuki T, Hirokane M, Matsuda Y, Sugioka S, Kotani J. The Parker Flex-Tip tube for nasotracheal intubation: the influence on nasal mucosal trauma. Anaesthesia 2010; 65: 8-11.

73. Xue FS, Xiong J, Yuan YJ, Wang Q. The Parker Flex-Tip tube for nasotracheal intubation. Anaesthesia 2010; 65: 417; author re- 
ply 417-8.

74. Earle R, Shanahan E, Vaghadia H, Sawka A, Tang R. Epistaxis during nasotracheal intubation: a randomized trial of the Parker Flex-Tip ${ }^{\text {tw }}$ nasal endotracheal tube with a posterior facing bevel versus a standard nasal RAE endotracheal tube. Can J Anaesth 2017; 64: 370-5.

75. Lee JH, Kim CH, Bahk JH, Park KS. The influence of endotracheal tube tip design on nasal trauma during nasotracheal intubation: Magill-tip versus Murphy-tip. Anesth Analg 2005; 101: 1226-9.

76. Collins SR. Direct and indirect laryngoscopy: equipment and techniques. Respir Care 2014; 59: 850-62; discussion 862-4.

77. Doherty JS, Froom SR, Gildersleve CD. Pediatric laryngoscopes and intubation aids old and new. Paediatr Anaesth 2009; 19 Suppl 1:30-7.

78. Kaplan MB, Hagberg CA, Ward DS, Brambrink A, Chhibber AK, Heidegger T, et al. Comparison of direct and video-assisted views of the larynx during routine intubation. J Clin Anesth 2006; 18: 357-62.

79. Rajendram R, Kale V. The use of advanced airway management devices in clinical practice. Eur J Anaesthesiol 2016; 33: 469-70.

80. Kido H, Komasawa N, Ishio J, Minami T. Use of a modified McGRATH MAC and tracheal tube introducer for difficult nasal intubation. J Clin Anesth 2016; 34: 255-6.

81. Arslan Zİ, Türkyılmaz N. Which nostril should be used for nasotracheal intubation with Airtraq $\mathrm{NT}^{\circ}$ : the right or left? A randomized clinical trial. Turk J Med Sci 2019; 49: 116-22.

82. Agrò F, Hung OR, Cataldo R, Carassiti M, Gherardi S. Lightwand intubation using the Trachlight: a brief review of current knowledge. Can J Anaesth 2001; 48: 592-9.

83. Manabe Y, Seto M, Iwamoto S, Tominaga S, Taniguchi S. The success rate of nasotracheal intubation using lightwand does not depend on the laryngoscopic view. J Anesth 2011; 25: 350-5.

84. Liberman H. A new intubating forceps. Anaesth Intensive Care 1978; 6: 162-3.

85. Sim L, Patel A, Enderby D. Modified Magill's forceps revisited. Anaesthesia 2004; 59: 97.
86. Boedeker BH, Bernhagen MA, Miller DJ, Doyle DJ. Comparison of the Magill forceps and the Boedeker (curved) intubation forceps for removal of a foreign body in a Manikin. J Clin Anesth 2012; 24: 25-7.

87. Munshey FN, Gamble JJ, McKay WP. Modified pediatric Magill forceps effect on nasal intubation time. Paediatr Anaesth 2016; 26: $221-2$.

88. Driver BE, Prekker ME, Klein LR, Reardon RF, Miner JR, Fagerstrom ET, et al. Effect of use of a bougie vs endotracheal tube and stylet on first-attempt intubation success among patients with difficult airways undergoing emergency intubation: a randomized clinical trial. JAMA 2018; 319: 2179-89.

89. Bączek M, Zagańczyk-Bączek M. Flexible tip bougie - the new introducer for intubation during cardiopulmonary resuscitation: a randomized crossover manikin study. Medicine (Baltimore) 2020; 99: e18452.

90. Seo KS, Kim JH, Yang SM, Kim HJ, Bahk JH, Yum KW. A new technique to reduce epistaxis and enhance navigability during nasotracheal intubation. Anesth Analg 2007; 105: 1420-4.

91. Elwood T, Stillions DM, Woo DW, Bradford HM, Ramamoorthy C. Nasotracheal intubation: a randomized trial of two methods. Anesthesiology 2002; 96: 51-3.

92. Watt S, Pickhardt D, Lerman J, Armstrong J, Creighton PR, Feldman L. Telescoping tracheal tubes into catheters minimizes epistaxis during nasotracheal intubation in children. Anesthesiology 2007; 106: 238-42.

93. Morimoto Y, Sugimura M, Hirose Y, Taki K, Niwa H. Nasotracheal intubation under curve-tipped suction catheter guidance reduces epistaxis. Can J Anaesth 2006; 53: 295-8.

94. Dougherty TB, Nguyen DT. Anesthetic management of the patient scheduled for head and neck cancer surgery. J Clin Anesth 1994; 6: 74-82.

95. Nagarkar R, Kokane G, Wagh A, Kulkarni N, Roy S, Tandale R, et al. Airway management techniques in head and neck cancer surgeries: a retrospective analysis. Oral Maxillofac Surg 2019; 23: 311-5. 